\title{
Elemental Analysis of Rare-earth Magnet Utilizing Cathodoluminescence
}

\author{
Susumu Imashuku ${ }^{1,2}$ and Jun Kawai ${ }^{1}$ \\ 1. Department of Materials Science and Engineering, Kyoto University, Kyoto, Japan. \\ 2. Institute for Materials Research, Tohoku University, Sendai, Japan.
}

Insulating materials and semiconductors emits light in the ultraviolet to infrared region by the bombardment of electrons. This phenomenon is called Cathodoluminescence (CL). CL phenomenon has been applied to analysis in many areas: an evaluation of defect distribution in semiconductor, an investigation of geological history of minerals, and so on. Especially, materials containing rare earth elements have high luminescence intensity. The detection limit of CL analysis is as low as ppb order. In the present study, we tried to apply CL analysis for evaluating rare earth elements in magnets. We also tried to sort magnets containing rare earth elements from their luminescent colors with a portable CL spectrometer we realized [1]. Analysis of rare earth elements in magnets is quite important for recycling due to high cost of rare earth elements.

Samples analyzed in the present study were a samarium-cobalt magnet and a neodymium magnet. We measured chloridized magnets because magnets themselves do not show CL phenomenon. The magnets were dissolved in 35\% hydrochloric acid $(\mathrm{HCl})$, and the solutions were dried in vacuum. Then, the vacuum-dried residues were measured with a SEM-CL system we customized. The SEM-CL system consisted of conventional SEM (JEOL, JSM-5610LVS), optical fiber, and optical spectrometer (Ocean optics, QE65Pro). The accelerating voltage and electron beam current of the SEM-CL system were set to $15 \mathrm{kV}$ and $2 \mu \mathrm{A}$, respectively. Measurement duration was set to 100 seconds. Pressure in a chamber of the SEM-CL system was about $10^{-3}$ Pa during the measurement.

A schematic view of the portable CL is shown in Fig. 1. + $\mathrm{z}$ plane of a single crystal of $\mathrm{LiTaO}_{3}$ with 6 $\mathrm{mm} \times 6 \mathrm{~mm}$ in $\mathrm{x}-\mathrm{y}$ plane and $5 \mathrm{~mm}$ in z-axis was attached on a Peltier device with silver paste. A gold wire was set in a needle holder. The needle holder was attached to the $\mathrm{LiTaO}_{3}$ crystal with silver paste. We captured CL images of the vacuum-dried residues through a glass viewport using a digital singlelens reflex camera equipped with a close-up lens with a focusing distance of $100 \mathrm{~mm}$. Exposure time of the camera was set to 100 sec. Measurements were carried out during cooling the $\mathrm{LiTaO}_{3}$ crystal after heating at $125^{\circ} \mathrm{C}$. Pressure of the sample chamber was set to $1 \mathrm{~Pa}$.

Figure 2 (a) shows CL spectrum of a vacuum-dried residue of the samarium-cobalt magnet alone with CL spectrum of samarium chloride $\left(\mathrm{SmCl}_{3}\right)$. Peak positions of the vacuum-dried residue of the samarium-cobalt magnet were the same as those of $\mathrm{SmCl}_{3}$. Figure 2 (b) shows CL spectrum of a vacuum-dried residue of the neodymium magnet. We confirmed that the neodymium magnet contained neodymium (Nd) (19.3\%), praseodymium (Pr) (4.8\%), dysprosium (Dy) (1.1\%), terbium (Tb) (2.3\%) by inductively-coupled plasma atomic emission spectrometry. By comparing a CL spectra of $\mathrm{NdCl}_{3}, \mathrm{PrCl}_{3}$, $\mathrm{DyCl}_{3}$, and $\mathrm{TbCl}_{3}$ as shown in figure 2 (b), peaks of the vacuum-dried residue of the neodymium magnet were indexed as shown in figure 2 (b). More than $1 \%$ of rare earth elements in the neodymium magnet were detected by SEM-CL analysis. Thus, we succeeded in detecting above $1 \%$ of rare earth elements in magnets by SEM-CL analysis by vacuum-drying solutions dissolved the magnets into $\mathrm{HCl}$.

We next tried to sort the magnets by CL images captured with the portable CL spectrometer. Figure 2 
(c) show CL image of the vacuum-dried residue of the samarium-cobalt magnet. It produced orange luminescence which corresponds to a color of the strongest peaks of the vacuum-dried residue of the samarium-cobalt magnet as shown in figure 2 (b). As for the vacuum-dried residue of the neodymium magnet, intensity was too low to detect its luminescence. We extracted iron ions from the solution the neodymium magnet dissolved using methyl isobutyl ketone [2] because the neodymium magnet contained $66 \%$ of iron. After iron ions were removed, the solution was vacuum-dried. The CL image of the vacuum-dried residue of the iron removed solution of the neodymium magnet was captured through a filter cut light with wavelengths less than $700 \mathrm{~nm}$. The vacuum-dried residue of the neodymium magnet produced red-purple color as shown in figure 2 (d). The red-purple color corresponds to illumination of $\mathrm{NdCl}_{3}$ (approximately $890 \mathrm{~nm}$ ) because the LED light produced a red-purple color when we captured an image of LED light with wavelength of 850 or $940 \mathrm{~nm}$ through the filter. Thus, the portable CL spectrometer can sort a samarium-cobalt magnet and a neodymium magnet by their luminescent colors.

References:

[1] S Imashuku, N Fuyuno, K Hanasaki and J Kawai, Rev. Sci. Instrum. 84 (2013), p. 126105.

[2] Y Kakita, Bunseki Kagaku, 16 (1967), p. 624.

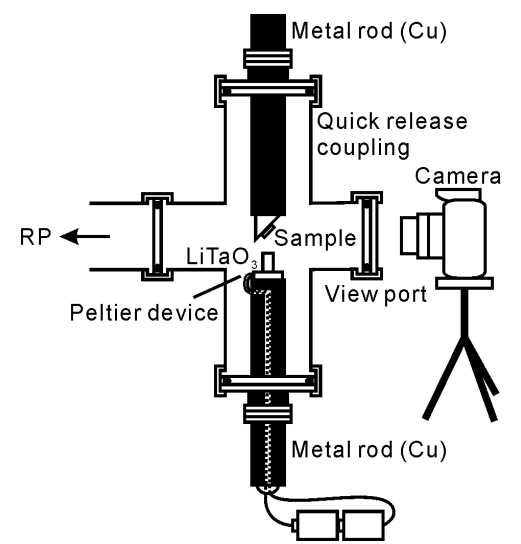

Figure 1. Schematic views of a portable CL spectrometer.
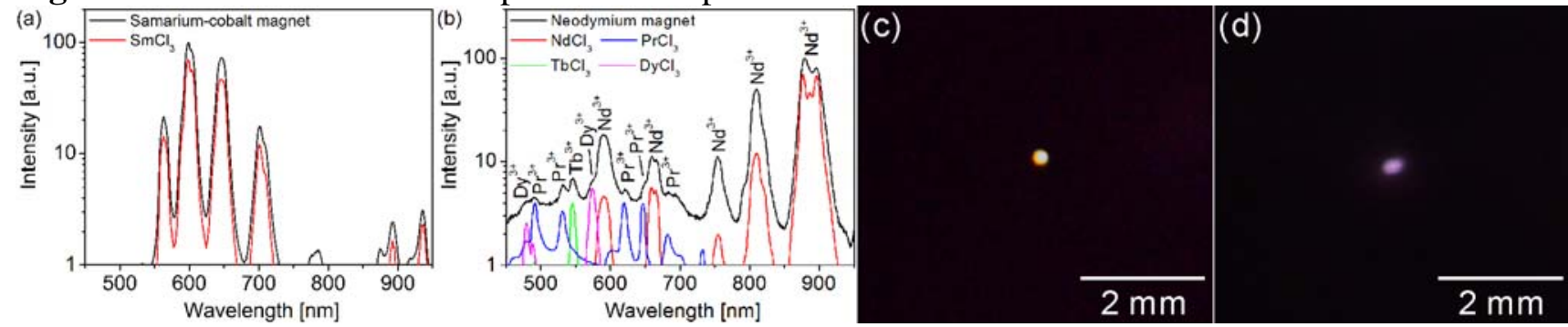

Figure 2. CL spectra of (a) a vacuum-dried residue of the samarium-cobalt magnet alone with $\mathrm{SmCl}_{3}$, and (b) a vacuum-dried residue of the neodymium magnet alone with $\mathrm{NdCl}_{3}, \mathrm{PrCl}_{3}, \mathrm{DyCl}_{3}$, and $\mathrm{TbCl}_{3}$ by SEM-CL analysis. CL images of (c) a vacuum-dried residue of the samarium-cobalt magnet and (d) a vacuum-dried residue of the iron removed solution of the neodymium magnet using a portable CL spectrometer. (d) was captured through a filter cut light with wavelengths less than $700 \mathrm{~nm}$. 